\title{
Satisfacción laboral de los profesionales sanitarios de un Hospital Universitario: análisis general y categorías laborales
}

\author{
César Carrillo-García ${ }^{1 *}$, María-Emilia Martínez-Roche², Carmen-Isabel Gómez-García y Mariano Meseguer-de-Pedro ${ }^{3}$ \\ 1 Escuela de Práctica Enfermera, Universidad de Murcia. Dirección General de Recursos Humanos del Servicio Murciano de Salud de Murcia (España) \\ 2 Departamento de Enfermería, Facultad de Enfermería, Universidad de Murcia (España) \\ 3 Departamento de Psiquiatría y Psicologia Social, Facultad de Psicologia, Universidad de Murcia (España)
}

\begin{abstract}
Resumen: La satisfacción laboral en los servicios de salud está implicada en la calidad asistencial, de ahí la importancia de su medición en los trabajadores de las instituciones sanitarias. El objetivo fue explorar y analizar el fenómeno de la satisfacción laboral en una muestra constituida por 546 profesionales sanitarios pertenecientes a un Hospital Universitario público. Se administró un cuestionario general que contenía un conjunto de preguntas de contenido sociodemográfico y se utilizó la Escala General de Satisfacción (Overall Job Satisfacción) adaptada y validada al castellano. Los resultados del estudio reflejan una satisfacción general media $(M=71.37 ; D T=$ 14.03), los "compañeros de trabajo" y el "superior inmediato" son los aspectos mejor evaluados. Los principales hallazgos reflejan diferencias importantes en los distintos niveles de satisfacción atendiendo a la categoría profesional, siendo los grupos de gestión y los médicos residentes lo más satisfechos, mientras que los facultativos especialistas de área, profesionales de enfermería y auxiliares de enfermería manifiestan niveles de satisfacción más reducidos.

Palabras clave: Satisfacción laboral; categorías profesionales; hospital uni-
\end{abstract} versitario; enfermería; auxiliar de enfermería; médicos residentes; médicos.

\section{Introducción}

Se ha señalado la importancia de la satisfacción laboral en los servicios de salud y las consecuencias que de ello se derivan, como son el aumento de la calidad asistencial de los servicios que prestan y la satisfacción de los usuarios. En esta línea se encuentran afirmaciones como las de Bravo (citado en Peiró, Silla, Sanz, Rodríguez y García, 2004, p.131) “...en los servicios de salud, la evaluación de la satisfacción laboral, está adquiriendo un creciente interés, ya que está relacionada con la satisfacción de los usuarios". La satisfacción laboral adquiere una especial relevancia en las organizaciones de servicios tal y como manifiestan Peiró et al. (2004, p.130):

La satisfacción laboral es un indicador del bienestar y la calidad de la vida laboral de los trabajadores. Su estudio es especialmente relevante en el ámbito de las organizaciones de servicios ya que influye en la calidad del servicio que presta.

Otros autores destacan la importancia de la motivación de las personas y su satisfacción con el trabajo, lo que revertirá en un aumento de la calidad del servicio:

Nadie duda en la actualidad de la importancia que tienen las personas en cualquier institución, ya sea pública o privada, para el logro y la consecución de objetivos. Es obvio que disponer de personas motivadas y satisfechas con su trabajo y con la organización aumenta su rendimiento y la calidad del servicio que prestan (Robles et al., 2005, p.128).

Es por lo que consideramos de vital valor el análisis de la satisfacción laboral ya que ésta va a repercutir directamente en el usuario tal y como muestran García, Ranchal, Biedma y

* Dirección para correspondencia [Correspondence address]:

César Carrillo García. Dirección General de Recursos Humanos. Servicio

Murciano de Salud. C/ Pinares No6. 4a Planta. Despacho 406, 30001. Murcia (España). E-mail: cesarcarrillo@um.es
Title: Job satisfaction of health professionals in a University Hospital: General analysis and job categories.

Abstract: Job satisfaction in health services is measured by the quality of care provided, hence the importance of surveying the workers of health institutions. The objective was to explore and analyze the phenomenon of the satisfaction of working in a sample made up of 546 health professionals belonging to a public University Hospital. Both a socio-demographic questionnaire and General Scale of Satisfaction (Overall Job Satisfacción) that were both validated in Spanish were given to the participants. The results of general satisfaction showed a mean of $71.37(S D=14.03)$. The subscales with better results were: colleagues and immediate superior. There were important differences in satisfaction scores between the different professional categories. The higher levels of general satisfaction were found in hospital management groups and resident doctors, while the lower levels of general satisfaction were found in medical area specialists, nurses and auxiliary nurses.

Key words: Job satisfaction; professional categories; university hospital; nursing; nursing assistant; resident physicians; physicians.

Serrano del Rosal (2008, p.1): "No sólo por lo que aportan en sí mismo, sino también porque se sabe que esta influye positivamente en su práctica laboral, incrementa la calidad del servicio y, finalmente en la satisfacción de los usuarios". También, la European Foundation for Quality Management (1999) considera que la satisfacción del profesional en el ámbito asistencial es uno de los elementos fundamentales para evaluar la calidad de una institución sanitaria.

La satisfacción de los profesionales sanitarios es un campo ampliamente explorado, evaluándose la satisfacción de todas las categorías profesionales en el ámbito de la salud. Bobbio y Ramos (2010), mostraron que los auxiliares de enfermería eran los más satisfechos y los facultativos los más insatisfechos; García, Luján y Martínez (2007) concluían que los enfermeros y las auxiliares eran los más satisfechos y los facultativos los profesionales más insatisfechos. Sin embargo, Martín, Ruiz y Sánchez (2005) y Robles et al. (2005) encontraron que el más insatisfecho era el colectivo de enfermería.

Nuestro estudio se enmarca en el marco teórico de la Teoría Bifactorial de Herzberg, cuyos pilares fundamentales son: que el trabajador posee dos tipos de necesidades, las higiénicas, que se refieren al medio ambiente físico y psicológico del trabajo (satisfacción extrínseca) y las motivacionales, que se refieren al contenido del trabajo (satisfacción intrínseca). En segundo lugar, si se satisfacen las necesidades higiénicas, el trabajador no está insatisfecho, pero tampoco satisfecho, se encuentra en un estado que se puede calificar como neutro. Y por último, el trabajador estará satisfecho en la medida en que se alcancen las necesidades de motivación. Por lo tanto, mejorando las condiciones higiénicas se puede 
hacer que la insatisfacción tienda hacia una posición neutra, y aumentando los factores motivacionales se pueda pasar de un estado neutro a un estado de satisfacción (ver en García y Meseguer, 2012).

El objetivo de esta investigación fue analizar el fenómeno de la satisfacción de la vida laboral de los profesionales sanitarios y describir los niveles de satisfacción laboral en los distintos grupos profesionales, entendiendo que puede haber diferencias significativas entre las categorías profesionales.

De acuerdo a la Teoría Bifactorial de Herzberg, hipótesis 1: el grupo que desempeña cargos de gestión estará más satisfecho que el resto de grupos profesionales. Este grupo de trabajadores, de libre designación, y dadas sus condiciones laborales más beneficiosas, así como el desempeño de tareas intrínsecamente más motivantes, su percepción de la satisfacción será mayor que la del resto de profesionales sanitarios.

El resto de hipótesis se plantean en la dirección que apuntan las investigaciones empíricas analizadas:

Hipótesis 2: los enfermeros serán posiblemente los profesionales más satisfechos.

Hipótesis 3: probablemente los facultativos será la categoría que muestre niveles de satisfacción más baja.

\section{Método}

\section{Diseño}

Para la realización de esta investigación se aplicó un diseño ex post facto y, en concreto, se realizó un estudio retrospectivo con un solo grupo mediante la aplicación de instrumentos de medición psicológica a una muestra de profesionales sanitarios (Montero y León, 2007).

\section{Participantes}

Para la realización del presente estudio se utilizó una muestra de conveniencia. Participaron voluntariamente 546 de los 1194 profesionales del Hospital Universitario pertenecientes a las categorías laborales de facultativos especialistas de área, médicos residentes, enfermeros y auxiliares de enfermería, igualmente se incluyó también a todos los profesionales de las anteriores categorías con cargos de responsabilidad en gestión de equipos y que estén integrados en la plantilla del citado hospital. El error muestral estimado (ET) se situaría en el $\pm 3.2 \%$ y un nivel de confianza del $95.5 \%$ ( $k$ =2). El porcentaje de participación fue del $45.72 \%$.

Comenzando por el género de los entrevistados y considerando todas las categorías laborales, predomina el género femenino con un $73.4 \%$ de la muestra. En cuanto a la edad, la mayoría de los profesionales entrevistados son de mediana edad, situándose entre 31 y 50 años, hallándose el 66.7\% de la muestra. En cuanto al perfil de características laborales, predominó la categoría profesional de enfermería (38.5\%) seguido por las de auxiliares de enfermería (28.8\%), los fa- cultativos especialistas de área (17.8\%), los médicos residentes $(5.9 \%)$ y los pertenecientes a las categorías de gestión ( $9 \%$ ); según el tipo de contrato, se registró un $42.5 \%$ de profesionales con plaza en propiedad, un $32.6 \%$ de interinos y un $24.9 \%$ de personal contratado. La mayor parte de los profesionales seguían un turno diurno $(48.2 \%)$, y un $32.6 \%$ un turno rodado con noches, el resto $(19.2 \%)$ tenían turnos de refuerzo o noches fijas. Por lo que se refiere a los años de antigüedad en el hospital, la media para el conjunto de los entrevistados se sitúa en 8.3 años $(D T=5.1)$

\section{Instrumentos de evaluación}

Se administró un cuestionario general que contenía un conjunto de preguntas de contenido sociodemográfico y una escala general de satisfacción. Para el estudio de la satisfacción de la vida laboral, se utilizó la Escala General de Satisfacción (Overall Job Satisfacción) desarrollada por War, Cook and Wall (1979) y adaptada y validada al castellano por Pérez y Fidalgo (1995). Esta escala solicita a los trabajadores el nivel de satisfacción con distintos elementos de la vida laboral, con un sistema de respuesta tipo Likert de siete puntos de anclaje, desde 1 (muy insatisfecho) hasta 7 (muy satisfecho). Los 15 ítems de la escala original están divididos en dos subescalas, una de factores intrínsecos, referentes al propio contenido del trabajo y que aborda aspectos como el reconocimiento obtenido por el trabajo, responsabilidad asignada, promoción profesional, aspectos relativos al contenido de la tarea, reconocimiento obtenido por el trabajo realizado, etc., esta escala está formada por siete ítems. Y otra de factores extrínsecos, vinculados con el contexto donde se desarrolla la actividad laboral que indaga sobre la satisfacción del trabajador con aspectos relativos a la organización del trabajo como el horario, la remuneración, las condiciones físicas del trabajo, relaciones entre dirección y trabajadores del hospital, la estabilidad laboral, etc., esta escala la constituyen ocho ítems. Esta escala de satisfacción laboral, es ampliamente usada y se encuentra insertada dentro del marco conceptual de la Teoría Bifactorial de Herzberg. La puntuación total se obtiene de la suma de los posicionamientos de los encuestados en cada uno de los ítems. La puntuación total de la escala oscila entre 15 y 105, de manera que una mayor puntuación refleja una mayor satisfacción general, respecto a las subescalas de satisfacción intrínseca y extrínseca, su corrección es idéntica a la de la escala general si bien, debido a su menor longitud, sus valores oscilan entre 7 - 49 para la satisfacción intrínseca y 8 - 56 para la satisfacción extrínseca (Pérez y Fidalgo, 1995). Mostró una consistencia interna según el $\alpha$ de Cronbach de .89.

\section{Procedimiento}

La muestra de profesionales se seleccionó de forma no aleatoria, la participación de los mismos fue de forma voluntaria, asegurando en todo momento la confidencialidad y el anonimato de los datos recogidos, lo que es garante de la va- 
lidez de las respuestas. Los cuestionarios se entregaron a los profesionales en sobre, para que una vez cumplimentados lo devolvieran cerrado, junto con una presentación exponiendo de forma general que se trataba de un estudio de satisfacción laboral.

La técnica de captación de información utilizada ha sido el cuestionario autoadministrado en el puesto de trabajo de los profesionales sanitarios, obteniéndose la información a través de la cumplimentación de los mismos, previamente codificados. Se informó al Gerente, Director Médico y Director de Enfermería; para seguidamente informar a los Jefes de Servicio y Supervisores de los distintos servicios, que a su vez fueron los que distribuyeron el cuestionario entre los profesionales sanitarios para su cumplimentación, siendo también ellos los encargados de su posterior recogida; los cuales la distribuyeron con una metodología, no solo rigurosa, sino también altruista y generosamente. El cuestionario se pasó entre los profesionales sanitarios del citado hospital durante el primer trimestre de 2010. Se repartieron un total de 1194 cuestionarios, de los cuales se devolvieron 546 (tasa de respuesta $45.72 \%$ ).

La presente investigación se realizó bajo el compromiso del cumplimiento de las normas éticas de investigación y de los requerimientos legales imprescindibles para poder realizar este estudio. De igual forma se respetó en todo momento la Ley Orgánica 15/1999, de 13 de diciembre, de Protección de Datos de Carácter Personal. Asimismo, se pidió autorización por escrito al Director Gerente del Hospital Universitario, para la realización de presente estudio, así como a los autores de la escala NTP 394: Satisfacción Laboral: escala general de satisfacción.

\section{Análisis de datos}

La explotación de los datos se analizó con el programa estadístico SPSS (v.15), que incluyen tanto marginales de respuesta como tablas de contingencia, estimando también las medias de tendencia central y las medidas de dispersión.

\section{Resultados}

\section{Análisis descriptivo}

En primer lugar analizamos el nivel medio de satisfacción considerando todas las categorías laborales y de acuerdo a la estructura original de la Escala General de Satisfacción, abordamos un nivel de análisis diferenciando entre satisfacción intrínseca, extrínseca y satisfacción general. Respecto a la satisfacción general, la media de los participantes se sitúa en $71.37(D T=14.03)$, por su parte, la satisfacción intrínseca es del $32.68(D T=7.84)$, en tanto que la obtenida en la satisfacción extrínseca se sitúa en 38.69 ( $D T=7.07)$.

En un análisis más pormenorizado de los 15 ítems que componen el cuestionario, la categoría de Gerencia puntúa por encima de los demás grupos profesionales en 11 de los 15 ítems (véase Tabla 1). En concreto los relacionados con elección del método de trabajo (ítem 2), responsabilidad asignada (ítem 6), utilización de capacidades personales (ítem 8), posibilidades de promoción (ítem 10), atención a las sugerencias que se presentan (ítem 12), condiciones físicas del trabajo (ítem 1), relación con los compañeros de trabajo (ítem 3), relaciones entre dirección y trabajadores (ítem 9), la manera en la que se gestiona (ítem 11), el horario de trabajo (ítem 13) y la estabilidad en el empleo (ítem 15). Por el contrario, el grupo que puntúa más bajo es el de los facultativos especialistas de área, en concreto en 9 de los 15 ítems.

Tabla 1. Valoraciones medias de los ítems de satisfacción.

\begin{tabular}{|c|c|c|c|c|c|c|}
\hline \multirow[t]{2}{*}{ Items } & \multicolumn{5}{|c|}{ Categoría } & \multirow[b]{2}{*}{$M$} \\
\hline & Gerencia & FEA & MIR & Enfermería & $\mathrm{AE}$ & \\
\hline P1. Condiciones físicas del trabajo & 4.7 & 4.1 & 4.6 & 4.2 & 4.3 & 4.26 \\
\hline P2. Libertad para elegir tu propio método de trabajo & 5.3 & 4.8 & 4.8 & 4.7 & 4.7 & 4.79 \\
\hline P3. Tus compañeros de trabajo & 5.9 & 5.9 & 5.9 & 5.9 & 5.8 & 5.87 \\
\hline P4. Reconocimiento que obtienes por el trabajo bien hecho & 4.9 & 4.3 & 5.2 & 4.4 & 4.3 & 4.44 \\
\hline P5. Tu superior inmediato & 5.5 & 5.4 & 5.8 & 5.8 & 5.7 & 5.67 \\
\hline P6. Responsabilidad que se te ha asignado & 5.6 & 5.1 & 5.1 & 5.2 & 5.2 & 5.20 \\
\hline P7. Tu salario & 4.8 & 4.9 & 5.5 & 5.0 & 3.9 & 4.67 \\
\hline P8. La posibilidad de utilizar tus capacidades & 5.3 & 4.6 & 5.1 & 4.8 & 4.7 & 4.79 \\
\hline P9. Relaciones entre dirección y trabajadores del hospital & 5.0 & 3.8 & 4.3 & 3.6 & 3.6 & 3.79 \\
\hline P10. Tus posibilidades de promocionar & 5.0 & 3.6 & 4.6 & 3.8 & 3.8 & 3.95 \\
\hline P11. El modo en el que el hospital está gestionado & 4.5 & 3.5 & 4.3 & 3.5 & 3.5 & 3.65 \\
\hline P12. La atención que se presta a las sugerencias que haces & 4.8 & 3.8 & 4.5 & 4.1 & 4.1 & 4.13 \\
\hline P13. Tu horario de trabajo & 5.6 & 5.2 & 4.8 & 5.1 & 5.6 & 5.32 \\
\hline P14. La variedad de las tareas que realizas en tu trabajo & 5.2 & 5.0 & 5.3 & 5.0 & 5.1 & 5.08 \\
\hline P15. Tu estabilidad en el empleo & 6.0 & 5.5 & 5.1 & 5.0 & 5.1 & 5.22 \\
\hline
\end{tabular}

Nota. FEA: facultativo especialista de área. MIR: médico interno residente. AE: auxiliar de enfermería. M: media.

Por otra parte, considerando la media general de los componentes de la satisfacción laboral, los mejores evaluados, con puntuaciones medias de 5 (moderadamente satisfe- cho) o superior son: los compañeros de trabajo $(M=5.87)$, el superior inmediato $(M=5.67)$, el horario de trabajo $(M=$ 5.32), la responsabilidad asignada $(M=5.20)$, la estabilidad 
en el empleo $(M=5.22)$ y la variedad de las tareas $(M=$ 5.08). Por el contrario, los componentes peor valorados: las posibilidades de promoción $(M=3.95)$ relaciones entre dirección y trabajadores del hospital $(M=3.79)$ y el modo en el que el hospital está gestionado $(M=3.65)$.

\section{Comprobación de las hipótesis}

Siguiendo la estructura original de la Escala General de Satisfacción y atendiendo a los resultados del estudio según las distintas categorías profesionales estudiadas, se pone de manifiesto que la categoría de gestión muestra el mayor nivel de satisfacción (78.06 de satisfacción general), confirmándose la primera hipótesis al ser los profesionales pertenecientes a dicha categoría los más satisfechos. Seguidamente los médicos residentes, obtienen un valor de satisfacción general de 74.97, no confirmándose la segunda hipótesis, al no ser los enfermeros los profesionales sanitarios asistenciales más satisfechos. Por su parte, enfermería obtiene un 70.60 y auxiliares de enfermería un 70.42, en tanto, que el grupo de facultativos especialistas de área presenta la puntuación más baja (69.99), confirmándose nuestra tercera hipótesis, (véase Tabla 2). Estas diferencias observadas entre categoría laboral y satisfacción son significativas, tanto en lo que se refiere a la satisfacción general $\left(\chi^{2}=15.05, p=.004\right)$, como a la satisfacción intrínseca $\left(\chi^{2}=13.85, p=.008\right)$ y a la extrínseca $\left(\chi^{2}\right.$ $=15.93, p=.003)$.

Tabla 2. Puntuación total y desviación típica $(D T)$ de las diferentes variables respecto a la categoría.

\begin{tabular}{|c|c|c|c|c|c|}
\hline & & & Satisfacción General & Satisfacción Intrínseca & Satisfacción Extrínseca \\
\hline \multirow{10}{*}{ 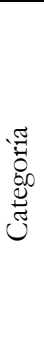 } & \multirow[t]{2}{*}{ Gerencia } & Puntuación total & 78.06 & 36.24 & 41.82 \\
\hline & & $D T$ & 11.19 & 5.81 & 6.18 \\
\hline & \multirow[t]{2}{*}{ FEA } & Puntuación total & 69.99 & 31.42 & 38.57 \\
\hline & & $D T$ & 13.68 & 7.43 & 7.23 \\
\hline & \multirow[t]{2}{*}{ MIR } & Puntuación total & 74.97 & 34.75 & 40.22 \\
\hline & & $D T$ & 10.37 & 6.43 & 4.74 \\
\hline & \multirow[t]{2}{*}{ Enfermero } & Puntuación total & 70.60 & 32.17 & 38.43 \\
\hline & & $D T$ & 14.48 & 8.28 & 7.04 \\
\hline & \multirow[t]{2}{*}{$\mathrm{AE}$} & Puntuación total & 70.42 & 32.59 & 37.84 \\
\hline & & $D T$ & 14.51 & 7.97 & 7.42 \\
\hline
\end{tabular}

Nota. DT: desviación típica. FEA: facultativo especialista de área. MIR: médico interno residente. AE: auxiliar de enfermería.

Para pormenorizar más las diferencias entre grupos profesionales, realizamos un análisis post-hoc de las distintas categorías que configuran la muestra, así en satisfacción general, la categoría de gerencia muestra diferencias estadísticamente significativas en comparación con los grupos de facultativos $\left(\chi^{2}=93.331, p=.007\right)$, enfermeros $\left(\chi^{2}=82.035, p=\right.$ .011) y auxiliares de enfermería $\left(\chi^{2}=88.010, p=.006\right)$, lo que muestra que el grupo de gerencia es el más satisfecho, no habiendo diferencias significativas entre el resto de los grupos profesionales. Respecto a la satisfacción extrínseca, la categoría de gerencia muestra diferencias significativas respecto a enfermería $\left(\chi^{2}=76.524, p=.022\right)$ y auxiliares de enfermería $\left(\chi^{2}=92.342, p=.003\right)$. Por último, respecto a la satisfacción intrínseca, la categoría de gerencia muestra diferencias estadísticamente significativas en comparación con los grupos de facultativos $\left(\chi^{2}=101.175, p=.002\right)$, enferme$\operatorname{ros}\left(\chi^{2}=77.419, p=.021\right)$ y auxiliares de enfermería $\left(\chi^{2}=\right.$ $72.364, p=.051)$.

\section{Discusión y Conclusiones}

El propósito de nuestra investigación fue analizar el fenómeno de la satisfacción de la vida laboral según la categoría laboral de los profesionales sanitarios. El primer dato de interés es que la puntuación media se sitúa en 71.37 , lo que podemos considerar como positivo y relevante, encontrán- donos en un intervalo muy similar a numerosos estudios (Bustos y Carrizosa, 2010; Fernández, 2002; García et al., 2007; Hernández et al., 2012; Herrera y Manrique, 2008; López, Bernal y Cánovas, 2001; López et al., 2011; Molina y Avalos, 2007; Parada et al., 2005; Ponce, Ponce y Lugo, 2009; Ponce, Reyes y Ponce, 2006; Sánchez, Torres y Palacio, 2010). Este resultado es especialmente significativo y demuestra que a pesar de la situación de crisis que se está viviendo, los profesionales pertenecientes a las profesiones relacionadas con el ámbito sanitario están mayoritariamente satisfechos con el ejercicio de su profesión. Con esto, la calidad asistencial, el trato dispensado y las buenas prácticas profesionales están respaldadas, tal y como manifiestan Saturno, Quintana y Varo (1997).

Un análisis más pormenorizado por ítems, nos permitirán señalar los elementos en los que se fundamenta esta satisfacción, así los dos aspectos mejor valorados han sido "los compañeros de trabajo" y "el superior inmediato". Las relaciones personales en el ámbito laboral pueden considerarse no solo como fuente de satisfacción sino como un importante factor amortiguador del desgaste profesional, quedando de manifiesto que el apoyo grupal tiene ciertas implicaciones de cara a la mejora de la calidad de vida laboral (Topa, Moriano y Morales, 2009). Estos dos aspectos, dentro de la teoría de Herzberg son elementos higiénicos que contribuyen a reducir la insatisfacción, pero no aumentar la satisfacción laboral. Igualmente, el "horario de trabajo" y la "es- 
tabilidad en el empleo" son factores valorados como muy positivos y dentro de la categoría de factores higiénicos. Y, el otro factor valorado como muy positivo dentro de los motivadores es "la responsabilidad asignada", que dentro de este entramado de organización burocrática-profesional, está más relacionado con un elemento extrínseco que intrínseco.

En el otro extremo, los tres aspectos que obtienen niveles de satisfacción negativos son las "posibilidades de promoción", las "relaciones entre dirección y trabajadores del hospital" y "el modo en que el hospital está gestionado". Evidentemente en la estructura organizativa antes comentada, las posibilidades de promoción se otorgan sobre todo por antigüedad, y no se valoran prácticamente otros méritos personales y profesionales. En cuanto a las "relaciones entre dirección y trabajadores del hospital" y "el modo en que el hospital está gestionado" suelen ser dimensiones que el trabajador considera muy politizadas y ajenas a la defensa de sus intereses profesionales, lo que hace que se mantenga como un elemento negativo.

En cuanto al análisis de la satisfacción entre las distintas categorías profesionales, es de destacar que la categoría más satisfecha es la del personal de gestión. Donde 11 de los 15 indicadores obtienen puntuaciones más altas que el resto de los grupos. Y, sobre todo, destacan los factores motivacionales como la autonomía para el ejercicio de su trabajo, el reconocimiento social, la responsabilidad, las posibilidades de promoción o la influencia que ejerce en la organización. Es de destacar, que estos puestos están ocupados por personas de libre designación, y que además de la satisfacción con el estatus conlleva mejoras en las condiciones de trabajo (turnicidad, nocturnidad, etc.) y en el sistema retributivo. Lo que hace que sea un colectivo especialmente satisfecho.

En cuanto a los grupos asistenciales, aunque las hipótesis de trabajo, extraídas de los distintos estudios analizados, partían de la consideración que enfermería era el colectivo más satisfecho y los facultativos el menos. En esta investigación, el haber incluido al personal médico residente ha descartado a la enfermería como el colectivo más satisfecho. El hecho de haber alcanzado recientemente el puesto, estar en formación retribuida del mismo, y de alto contenido vocacional, podría explicar esa satisfacción tanto extrínseca como intrínseca de este colectivo sobre el resto.

\section{Referencias}

Bobbio, L. y Ramos, W. (2010). Satisfacción laboral y factores asociados en personal asistencial médico y no médico de un hospital nacional de Lima-Perú. Revista Peruana de Epidemiología, 14(2), 133-138.

Bustos, R. y Carrizosa, M. D. (2010). Satisfacción laboral de enfermería en unidades de hospitalización médico-quirúrgica del Complejo Hospitalario de Albacete. Revista de Administración Sanitaria, 1(3). Recuperado de http://www.opinionras.com/files/Originales_e_RAS_Articulo_3_2010 .pdf

European Foundation for Quality Management. (1999). Modelo Europeo de Excelencia. Madrid: Club Gestión de la Calidad.
En relación al puesto de enfermero/a, nuestro trabajo, al igual que otros (García, Moro y Medina, 2010; Ponce et al., 2009), asimila la satisfacción con el personal auxiliar y más satisfechos que con el colectivo de facultativos. Este grupo profesional, reiteradamente, aparece como el más insatisfecho. Posiblemente, la sobrecarga de trabajo, las relaciones profesionales con otros grupos asistenciales, las quejas sobre el salario, las pocas relaciones sociales con iguales, el trabajo emocional donde se tiende a evitar emociones negativas, etc., estén posiblemente en la base de la falta de satisfacción de este colectivo.

En resumen, la ausencia de elementos motivadores y la falta de satisfacción de los facultativos son elementos de consideración. El diseño de acciones de mejora por parte de la dirección para corregir aquellos aspectos con peores resultados debe ser una actividad habitual ya que como afirman Ríos, Godoy y Sánchez (2011), establecer mecanismos o ciclos de mejora debe ser prioritario para cualquier equipo directivo. Convirtiéndose además en un área de investigaciónacción, donde la satisfacción laboral puede ser un eje en el que se evalúen las prácticas organizacionales.

En cuanto a las limitaciones de nuestra investigación es preciso matizar en primer lugar, y por las dificultades obvias, tuvimos que disponer de una muestra de profesionales seleccionada de forma no aleatoria, que participaron de forma voluntaria en el estudio, los datos fueron recogidos mediante cuestionario, quedando así a criterio de los participantes la sinceridad de las respuestas.

De modo resumido, los principales hallazgos reflejan que las diferencias observadas en los distintos niveles de satisfacción atendiendo a la categoría profesional sugieren que, para la mayor parte de los aspectos en análisis, el grupo de gestión presenta los niveles más elevados de satisfacción, seguido por el de los médicos residentes. Frente a estos grupos, los facultativos especialistas de área, enfermería y auxiliares de enfermería, destacarían por ser los que ofrecen niveles de satisfacción más reducidos, debido creemos en el caso de enfermería, por ocupar un puesto estratégico entre los facultativos y pacientes, y ser el profesional que más tiempo pasa en contacto con el usuario, es por lo que puede recaer en ellos la responsabilidad de filtrar, canalizar y solucionar numerosos dilemas que pueden surgir durante la actividad asistencial en el hospital.

Fernández, M. I. (2002). Satisfacción laboral en los profesionales de la salud. Revista Virtual de Odontología. Ejercicio Profesional, 3(32). Recuperado de http://www.odontomarketing.com/art71 dic2002.htm

García, A., Moro, M. N. y Medina M. (2010). Evaluación y dimensiones que definen el clima y la satisfacción laboral en el personal de enfermería. Revista de Calidad Asistencial, 25(4), 207-214. Doi: 10.1016/j.cali.2010.02.003

García, J. M., Ranchal, J., Biedma, L. y Serrano del Rosal, R. (2008). Preferencias y expectativas de los médicos residentes en Andalucía. (Documentos de trabajo). Instituto de Estudios Sociales Avanzados. Recuperado de http://digital.csic.es/bitstream/10261/24225/1/Preferencias $\% 20 y \% 20$ expectativas.pdf 
García, M. y Meseguer, M. (2012). Análisis individual del comportamiento humano en las organizaciones. En M. Meseguer y M. I. Soler (Eds.), Psicología del trabajo y de las organizaciones (pp. 45-73). Murcia: Diego Marín.

García, M., Luján, M. E. y Martínez, M. A. (2007). Satisfacción laboral del personal de salud. Revista de Enfermería del Instituto Mexicano del Seguro Social, 15(2), 63-72.

Hernández, M., Hernández, A., Nava, M. G., Pérez, M. T., Hernández, M. G., Matus, R. y Balseiro, C. (2012). Satisfacción laboral del profesional de enfermería en cuatro instituciones de salud. Enfermería Universitaria ENEO-UNAM, 9(1), 7-15.

Herrera, G. y Manrique, F. G. (2008). Condiciones laborales y grado de satisfacción de profesionales de enfermería. Aquichan, 8(2), 243-256.

López, F., Bernal, L. y Cánovas, A. (2001). Satisfacción laboral de los profesionales de un hospital comarcal de Murcia. Revista de Calidad Asistencial, 16, 243-246.

López, M. P., Torrejón, G., Martín, A., Martín, J., Pleite, F. y Torres, M. A. (2011). Estrés y satisfacción laboral de las enfermeras de hospitales toledanos. Metas de Enfermería, 14(8), 8-14.

Martín, M., Ruiz, V. y Sánchez, M. (2005). Satisfacción laboral en el personal de las fundaciones sanitarias en Galicia. Revista de Administración Sanitaria, 3(2), 303-313

Molina, J. M. y Avalos, F. (2007). Satisfacción laboral y desgaste profesional de personal de enfermería. Revista Tesela, 1. Recuperado de http://www.index-f.com/tesela/ts1/ts6327r.php

Montero, I. y León, O. G. (2007). A guide for naming research studies in Psychology. International Journal of Clinical and Health Psicology, 7(3), 847862.

Parada, M. E., Moreno, R., Mejías, Z., Rivas, A., Rivas, F., Cerrada, J. y Rivas, F. (2005). Satisfacción laboral y síndrome de burnout en el personal de Enfermería de Instituto Autónomo Hospital Universitario Los Andes (IAHULA), Mérida, Venezuela, 2005. Revista de la Facultad Nacional de Salud Pública, 23(1), 33-45.

Peiró, J. M., Silla, I., Sanz, T., Rodríguez, J. y García, J. L. (2004). Satisfacción laboral de los profesionales de Atención Primaria. Psiquis: Revista de Psiquiatría, Psicología Médica y Psicosomática, 25(4), 129-140.
Pérez, J. y Fidalgo, M. (1995). NTP: 394: Satisfacción laboral: escala general de satisfacción. (Informe NTP: 394). Instituto Nacional de Seguridad e Higiene en el Trabajo. Recuperado de http://www.insht.es/InshtWeb/Contenidos/Documentacion/FichasT ecnicas/NTP/Ficheros/301a400/ntp_394.pdf

Ponce, J., Ponce, G. y Lugo, I. (2009). Satisfacción laboral de personal de Enfermería en un hospital de gineco-obstetricia del Instituto Mexicano del Seguro Social. Revista de Enfermería del Instituto Mexicano del Seguro Social, 17(2), 103-108.

Ponce, J., Reyes, H. y Ponce, G. (2006). Satisfacción laboral y calidad de atención de Enfermería en una unidad médica de alta especialidad. Revista de Enfermería del Instituto Mexicano del Seguro Social, 14(2), 65-73.

Ríos, M. I., Godoy, C. y Sánchez, J. (2011). Síndrome de quemarse por el trabajo, personalidad resistente y malestar psicológico en personal de enfermería. Anales de Psicología 27(1), 71-79.

Robles, M., Dierssen, T., Martínez, E., Herrera, P., Díaz, A. R. y Llorca, J. (2005). Variables relacionadas con la satisfacción laboral: un estudio transversal a partir del modelo EFQM. Gaceta Sanitaria, 19(2), 127-134.

Sánchez, S. Torres, M. D. y Palacio, R. (2010). Grado de satisfacción de las enfermeras del área de hospitalización quirúrgica de un hospital de nueva apertura en la Comunidad de Madrid. ENE, Revista de Enfermería, 4(1), 8-19. Recuperado de http://eneenfermeria.org/ojs/index.php/ENE/article/view/105/88

Saturno, P. J., Quintana, O. y Varo, J. (1997). ¿Qué es la calidad? En P. J. Saturno, J. J. Gascón y P. Parra (Eds.), Tratado de Calidad Asistencial en Atención Primaria. (pp.19-45). Madrid: Dupont Pharma.

Topa, G., Moriano, J. A. y Morales, J. F. (2009). Acoso laboral entre profesionales de enfermería: El papel protector de la identidad social en el trabajo. Anales de Psicología, 25(2), 266-276.

Warr, T., Cook, P. \& Wall, K. (1979). Scales for the measurement of some work attitudes and aspects of psychological well-being. Journal of Occupational Psychology, 52, 129-148.

(Articulo recibido: 28-02-2013; revisado: 03-01-2014; aceptado: 13-03-2014) 